\title{
Tendencias de investigación en bibliotecología y ciencia de la información en la Argentina durante las primeras décadas del siglo XXI
}

\section{Research trends in library and information science in Argentina during the first decades of the XXI century}

\author{
María Cecilia Corda**, ${ }^{*}$ Gustavo Liberatore ${ }^{2}$
}

Cómo citar (APA): Corda, M.C.; \& Liberatore, G. (2021). Tendencias de investigación en bibliotecología y ciencia de la información en la Argentina durante las primeras décadas del siglo XXI.

Iberoamerican Journal of Science Measurement and

Communication; 1(2), 1-19. https://

doi.org/ 10.47909/ijsmc.44

Recibido: 15-02-2021

Aceptado: 26-04-2021

Editor: Carlos Alberto Ávila Araújo

Copyright: () 2021 Corda \& Liberatore. This is an open access article distributed under the terms of the CC BY-NC 4.0 license which permits copying and redistributing the material in any medium or format, adapting, transforming and building upon the material as long as the license terms are followed.

\section{RESUMEN}

Objetivo. El propósito de este artículo es describir y analizar las líneas de investigación en Bibliotecología y Ciencia de la Información desarrolladas en los marcos institucionales de las universidades que dictan la carrera de bibliotecología, archivología y museología en la Argentina.

Diseño/Metodología/Enfoque. Se recuperaron los documentos que han sido presentados en los encuentros de docentes e investigadores de las escuelas del Mercosur, así como estudios anteriores que abordaban la problemática. Además, se sistematizaron las producciones de los docentes investigadores del campo disciplinar, utilizando la aplicación Publish or Perish, la cual toma a Google Académico como fuente de datos.

Resultados/Discusión. Se observó una consolidación del campo temático, tanto desde los aportes para el fortalecimiento conceptual y metodológico de la disciplina, como desde las indagaciones que pretenden resolver cuestiones que se suscitan en el campo profesional de bibliotecas, archivos, museos y otras unidades de información.

Conclusiones. Se destacó la existencia de ejes de trabajo que combinan temáticas tratadas a nivel internacional en la materia, así como áreas de especialización enfocadas en

1 Instituto de Investigaciones en Humanidades y Ciencias Sociales - IdIHCS (UNLP-CONICET), Universidad Nacional de la Plata; Instituto de Investigaciones Sociales de América Latina - IICSAL, (FLACSO-CONICET), Argentina.

*Autora correspondiente, email: mcorda@fahce.unlp.edu.ar; mccorda@flacso.org.ar. ORCID: 0000-0003-1885-7785.

2 Universidad Nacional de Mar del Plata. Departamento de Ciencia de la Información - CECID, Argentina.

Email:gliberat@mdp.edu.ar. ORCID: 0000-0002-5841-8739. 
realidades locales. Se demostró que el desarrollo y productividad investigativa se concentran en pocas escuelas. Finalmente, se abordó la incipiente constitución de los estudios de posgrado a nivel de especialización, maestría y doctorado que, con el tiempo, ampliará el espectro de investigaciones realizadas en las universidades que desarrollan estos tramos formativos.

Originalidad/Valor. No existía un estudio de este tipo en el que se combinaran el análisis documental con la bibliometría para develar el entramado de investigadores, temas y producción científica en Bibliotecología y Ciencia de la Información en la Argentina, no siempre sencillo de captar y abordar.

Palabras clave: Investigación en ciencias de la información; Formación bibliotecológica; Posgrado; Bibliometría; Producción científica; Argentina

\section{ABSTRACT}

Objective. This paper aims at describing and analyzing the research lines on Library and Information Science (LIS) developed within the institutional framework of the Argentinian universities where careers in Library Science, Archival Science, and Museology are offered.

Design/Methodology/Approach. Documents presented at academic events from the Mercosur's schools were gathered, as well as former studies that address the issue. Additionally, the scientific production of researchers and academicians from the LIS field was systematized using the Publish or Perish app, considering Google Scholar as a data source. Results/Discussion. A consolidation of the LIS field was observed, both from the contributions for the conceptual and methodological strengthening of the discipline, and from the inquiries that seek to resolve issues that arise in the professional field of libraries, archives, museums, and other information units.

Conclusions. The existence of work lines that combine topics dealt with at an international level in the field was highlighted, as well as areas of specialization, focused on local realities. It was shown that the research development and productivity are concentrated in few schools. Finally, the incipient constitution of postgraduate studies at the specialization, master's degree, and doctorate levels was addressed, which, over time, will broaden the spectrum of research carried out in the universities developing these training sections.

Originality/Value. There was no study of this type in which documentary analysis was combined with bibliometrics to reveal researchers, topics, and scientific production in the LIS field in Argentina, something not always easy to capture and address.

Keywords: Information science research; Library training; Postgraduate studies; Bibliometrics; Scientific Production; Argentina

\section{INTRODUCCIÓN}

El campo de la bibliotecología y ciencia de la información (ByCl) en Argentina se desenvuelve a través del dictado de las carreras relacionadas a nivel de grado y el desarrollo de líneas de investigación en diez carreras ofrecidas en universidades, aunque dichos desarrollos no son homogéneos. La primera carrera nació en el marco de la Universidad de Buenos Aires (UBA) en el año 1922. Luego surgió una propuesta formativa, aunque con sus vaivenes iniciales, en la Universidad Nacional de La Plata (UNLP), cuya fecha temprana de nacimiento data de 1949. Posteriormente, otras carreras emergieron en la Universidad Nacional del Nordeste (UNNE) en 1959, la Universidad Nacional de Córdoba (UNC) en 1960, la Universidad Nacional de Misiones (UNaM) en 1979, y la Universidad Nacional de Mar del Plata (UNMDP) 
en 1980. Más recientemente, en la Universidad Nacional del Litoral (UNL), la Universidad Nacional de La Rioja (UNLaR) y la Universidad Autónoma de Entre Ríos (UADER). Existen también dos carreras con modalidad a distancia en el contexto de universidades privadas, siendo una la de la Universidad Católica Argentina - Sede Paraná y la otra la de la Universidad Católica de Santiago del Estero. Finalmente, existió hasta 2010 una propuesta de formación en $\mathrm{ByCl}$ que se constituyó en la primera del ámbito privado dictada por la Universidad del Museo Social Argentino y que fue creada en 1931.

La larga tradición existente en nuestro país en la formación de profesionales en el campo de la $\mathrm{ByCl}$ no guarda equivalencias con el desarrollo de la investigación y la generación de recursos humanos volcados a esta dimensión. Desde una mirada integral, el ejercicio de la investigación en esta disciplina es un territorio poco explorado y débilmente estudiado en Argentina. Desde las propuestas formativas ligadas fuertemente a un perfil profesionalista hasta la débil inserción de la disciplina en el sistema científico/académico nacional, existe un buen número de razones acerca de por qué la $\mathrm{ByCl}$ constituye un campo de conocimiento periférico en términos de su estatus científico y su caudal investigativo.

La cuestión aquí planteada ha de rastrear sus orígenes en los niveles de formación de grado, tal como lo han estudiado Miguel, Salomoni e Hidalgo (2014), al recopilar y analizar los contenidos de investigación en los planes de estudio de las carreras en las universidades nacionales. En dicho trabajo, llegan a la conclusión de que hay pocos tópicos específicos referidos al conocimiento del estado de la investigación en $\mathrm{ByCl}$, problemática a la que el presente artículo pretende dar algunas respuestas. Asimismo, los autores se preguntan si la incorporación de asignaturas para la formación en investigación en las carreras de grado en el país ha contribuido a ampliar la comunidad de investigadores que ejercen dicha actividad; ya sea en la academia o en el ámbito profesional de las bibliotecas y centros de documentación, con una comunicación adecuada de resultados a través de publicaciones y otros típicos circuitos de comunicación científica.

En este marco, el presente trabajo aborda la investigación en $\mathrm{ByCl}$ desde una doble vertiente. Por un lado, se presenta una descripción del contexto de la disciplina en la Argentina a partir de los marcos institucionales desde donde se desarrollan las actividades de investigación, los principales antecedentes de aquellos estudios que han realizado aportes sobre el particular y el panorama existente en la actualidad en la formación de posgrado a nivel local. Por otro, y de manera complementaria, se realiza una aproximación empírica a partir de la recuperación y análisis de la producción científica de los últimos veinte años (2001-2020) de los docentes-investigadores que integran las escuelas universitarias. Esto se hizo con el fin de caracterizar y delimitar, por medio de la aplicación de indicadores bibliométricos, la evolución y situación actual de la disciplina en el contexto académico.

\section{Antecedentes}

\subsection{Investigación en bibliotecología y ciencias de la información a nivel nacional}

Una de las dificultades que atraviesa el campo de $\mathrm{ByCl}$ en la Argentina es la ausencia de instituciones intermedias, más allá de las universidades en las que se dicta la carrera, que visibilicen la producción y breguen por mejores condiciones de la investigación en dicho campo. Son varios los ejemplos que se pueden tomar de los modelos de institucionalización disciplinar vigentes, aunque el más próximo y consolidado a nivel regional es Brasil. Allí existe un entramado de estamentos a nivel nacional de los que carece nuestro país, como el Instituto Brasileiro de Informação em Ciência e Tecnologia (IBICT), la Associação Nacional de Pesquisa e Pós-Graduação em Ciência da Informação (ANCIB) y la Associação Brasileira 
de Educação em Ciência da Informação (Pinheiro y Loureiro, 2004; Valentim y Araujo, 2019).

En nuestra realidad nacional sólo existen dos entidades orientadas específicamente al campo de la investigación surgidas en ambientes académicos. Una de ellas es el Instituto de Investigaciones Bibliotecológicas (INIBI) ${ }^{1}$ de la UBA, creado en 1967 (Parada, 2017), pionero en la generación de líneas de investigación y cuadros científicos en la disciplina. Por su parte, en la UNMDP se ha puesto en funcionamiento a partir de 2017 el Centro de Estudios en Ciencia de la Información y Documentación (CECID) ${ }^{2}$, en donde confluyen los proyectos de investigación en curso.

En el ámbito de las publicaciones nacionales en $\mathrm{ByCl}$ existen en la actualidad tres revistas de perfil científico editas por instituciones académicas. La de mayor trayectoria es la revista Información, Cultura y Sociedad surgida en 1999 y editada por el INIBI. Le sigue la publicación Palabra clave [La Plata], creada en 2010 y editada por el Departamento de Bibliotecología de la UNLP, y la revista Prefacio iniciada en 2017 y gestionada por la Escuela de Bibliotecología de la UNC.

A nivel de las escuelas de $\mathrm{ByCl}$, existen cuatro espacios de discusión y difusión de la actividad investigadora. El primero de ellos surge por medio de las Jornadas de Intercambio y Reflexión acerca de la Investigación en Bibliotecología (JIRIB), organizadas desde el Departamento de Bibliotecología de la UNLP, las cuales se vienen realizando desde el año 2010. Son espacios de encuentro para la comunicación y el debate de proyectos; esto es, avances y resultados de investigaciones vinculados a la $\mathrm{ByCl}$. Surgieron luego de unas Jornadas previas realizadas en 2009 en conmemoración de los 60 años de la carrera de bibliotecología en la UNLP (Miguel, 2015). Además, el Departamento de Ciencia de la Información de la UNMDP organiza cada dos años las Jornadas Internas de Investigación en Bibliotecología desde el 2013, como espacio de socialización académica de las producciones generadas en este ámbito.

EI INIBI suele propiciar de modo regular ciclos de conferencias y encuentros que exponen los resultados de los proyectos en curso. ${ }^{3}$ Otro espacio de intercambio y desarrollo del campo se ha dado en los encuentros Mercosur. Según refiere Elsa E. Barber (2007), los mismos fueron originalmente planificados a partir de las ideas intercambiadas entre la presidenta y la secretaria ejecutiva de la Associação Brasileira de Escolas de Biblioteconomia e Documentação (ABEBD), Profesoras Jussara Pereira Santos e lara Conceicao Bitencourt Neves, y la entonces directora del Departamento de Bibliotecología y Documentación de la UBA, la misma Barber, en abril de 1996. Efectivamente, el primero se realizó en Porto Alegre en el año 1996. El último estaba planificado para llevarse a cabo en Montevideo, pero por motivo de la pandemia del COVID-19, se demoró su realización en el año 2020.

En dichos encuentros, que se extienden no sin dificultades hasta el día de hoy, formaron parte en algunas de sus ediciones Chile y Venezuela, además de los países que conforman estrictamente el Mercosur. Si bien los temas que se abordan se vinculan más a cuestiones curriculares de las carreras, no faltan reflexiones sobre investigación en el campo, donde suele persistir una mesa de discusión con relación a ella a lo largo de las sucesivas ediciones. También hay ocasiones en las que se llama a presentación de ponencias y se contribuye al debate desde ese tipo de aportes. Una dimensión que se encuentra más diluida en estos encuentros es la de la extensión universitaria, situación tal vez atribuible a que el desarrollo en la materia es muy disímil en las escuelas que suelen participar de la instancia.

La estructura de estas reuniones generalmente se replica y consiste en: 
- designación de la sede y la delineación de la temática convocante;

- organización de los informes por escuelas por países;

- tratamiento de dichos informes en la reunión de directores;

- discusión de docentes-investigadores en las seis áreas temáticas (que con más o menos variantes son las siguientes: fundamentos teóricos de la bibliotecología y la ciencia de la información; procesamiento de la información; recursos y servicios de información; tecnología de la información; gestión de la información y el conocimiento; investigación);

- ponencias y mesas de debate;

- conclusiones y recomendaciones; y

- designación de la nueva sede y temática convocante.

De este modo los encuentros facilitan, a través de los informes de las escuelas, un estado de situación sobre temas docentes y de investigación. A nivel local, las escuelas de bibliotecología en Argentina han procurado organizar una jornada con un año de anticipación a la realización de los encuentros de Mercosur. Las mismas se titulan: Jornadas de Docentes Investigadores Universitarios en Ciencia de la Información, conocidas como Jornadas DUCI, llevándose a cabo tres ediciones: una en Buenos Aires (2015), otra en Mar del Plata (2018) y la última en Posadas (2019), organizadas respectivamente por la UBA, la UNMDP y la UNaM.

Otro aporte en el eje de investigación es el que propició el Centro Argentino de Información Científica y Tecnológica (CAICyT), dependiente del Consejo Nacional de Investigaciones Científicas y Técnicas (CONICET), entidad que a partir del año 2012 se convirtió también en un centro de investigación, además de continuar siendo uno de servicios como tradicionalmente lo era. El laboratorio creado produce fuentes de datos y ofrece tecnologías en forma de infraestructuras de información (Bosch, 2015). Un antecedente al presente trabajo es el Proyecto Actividades de Investigación en Bibliotecología y Ciencias de la Información AICIB, que pretendía recabar la información sobre investigaciones, investigadores y producciones académicas en el campo de $\mathrm{ByCl}$. No obstante, debido a los vaivenes políticos y de gestión, dicho centro ha atravesado diversas vicisitudes en los últimos tiempos, lo que le ha impedido avanzar en estas líneas y/o articularse con las universidades en donde se imparte la carrera.

\subsection{Aproximaciones a la investigación en $\mathrm{ByCl}$}

Como se menciona en los párrafos introductorios, existen pocos registros de trabajos que se hayan desarrollado en procura de establecer la situación de la investigación dentro del campo disciplinar en la Argentina. En general, los antecedentes indican que la mayoría de los abordajes a esta problemática se han basado en modelos empíricos de análisis de la práctica científica, enfocados a espacios institucionales concretos o en la participación de investigadores en ciertas áreas de producción. En esta línea, Liberatore et al. (2015) indagan, en el contexto de la producción científica en humanidades y ciencias sociales de la UNMDP, en los patrones de publicación y difusión, con especial atención al campo de la ByCl. Entre los resultados observados se destacan aspectos relativos a los formatos de publicación, el marcado localismo de sus publicaciones como una respuesta lógica a la naturaleza de su 
objeto de estudio, y la lengua española como idioma de publicación en la mayoría de sus producciones. Se verifica además que la circulación se encuentra fuertemente restringida a fuentes nacionales, con reducida presencia de la producción a nivel regional e internacional, lo cual afecta negativamente la visibilidad y el impacto de la actividad investigadora. En el mismo sentido, Romanos y Contardi (2010) arriban a resultados similares respecto de las producciones de los docentes-investigadores de la Facultad de Filosofía y Letras de la UBA.

Los aportes más concretos a los procesos y dinámicas de la investigación en ByCl han sido generados en el ámbito del Departamento de Bibliotecología en la UNLP. Miguel (2009) sostiene que entre 1994 y 1999 la dirección de los proyectos de investigación estuvo a cargo de docentes investigadores formados en otras disciplinas y externos al propio Departamento y que a partir del año 2000, esa situación se modificó, como resultado de un crecimiento del plantel docente investigador que le permitió acceder a las categorías requeridas para la dirección o co-dirección de proyectos. En esta misma línea, González y Miguel (2010) y González, Miguel y Chinchilla Rodríguez (2010) revisaron la composición y la dinámica de los grupos de investigación entre los años 2000 y 2009, empleando técnicas bibliométricas y de análisis de redes sociales. El estudio, que es probablemente el antecedente más directo de esta investigación, estuvo orientado a una demarcación y análisis de los grupos en perspectiva comparada entre los proyectos y las coautorías. En dichos trabajos concluyeron que la permanencia de docentes-investigadores a lo largo del tiempo se vincula más con quienes desempeñan funciones de dirección de proyectos, no así con figuras más esporádicas que no terminan integrándose más tarde a las investigaciones que prosiguen. También señalaron los esfuerzos por construir grupos que no sean endogámicos y que se vinculen con otras universidades, además de propiciar enfoques interdisciplinarios de las líneas de indagación. Enfatizan, además, que la elección de las temáticas deben hacerse desde una perspectiva de la proyección internacional del trabajo investigativo desarrollado en el campo disciplinar. En este sentido, destacan el fortalecimiento y consolidación de ciertas líneas de investigación en la UNLP como es el caso de los estudios métricos de la información (Miguel, González y Archuby, 2010). Finalmente, Miguel et al. (2012) indagan sobre la articulación entre formación e investigación, reflexionando sobre la necesidad de acercar los problemas de la profesión a la investigación realizada en la academia, además de procurar más asignaturas involucradas con los procesos investigativos.

Dentro de los antecedentes, cabe mencionar también estudios orientados a explorar la inserción de investigadores del campo de la $\mathrm{ByCl}$ en áreas temáticas de aplicación transversal a múltiples campos científicos. Tal es el caso del trabajo realizado por Miguel y Dimitri (2013), en el que indagan sobre la producción científica publicada sobre estudios métricos en la Argentina dentro del periodo 1984-2012. En él se destaca que esta área de conocimiento, propia de la $\mathrm{ByCl}$, excede largamente en su autoría y aplicación a nuestro campo disciplinar. Aunque se observa una alta participación de profesionales de nuestro campo en los equipos de investigación que han desarrollado estudios en esta temática, se concluye que, dado su carácter multidisciplinar de aplicación, es un ámbito de confluencia de investigadores provenientes de otras áreas, particularmente de las ciencias naturales y exactas, ciencias de la salud, ciencias sociales, humanidades, ingenierías y tecnologías.

\subsection{Posgrados: diálogos posibles con líneas de investigación}

En lo que respecta a la formación de posgrado, durante largas décadas hubo un "área de vacancia" en el campo de la $\mathrm{ByCl}$ en Argentina; es decir, no existían programas universitarios (especializaciones, maestrías y doctorados) propuestos por las escuelas universitarias, con recursos humanos propios y perfiles adecuados a la realidad nacional (Liberatore, 2002, 
2011). Ante esta situación, algunas personas optaron por formarse en universidades extranjeras, o bien hacerlo en áreas temáticas relacionadas. Sin embargo, otras quedaron sin posibilidad de hacerlo, revistiendo solo el nivel de licenciatura o profesorado para ejercer sus funciones en el ámbito universitario. Se señala especialmente el problema de la carencia de formación del posgrado dado que es en este nivel donde un investigador comienza su formación como tal en cualquier campo científico, especialmente aquellos orientados a la obtención del doctorado. La consecuencia más visible de esta situación es que la investigación en $\mathrm{ByCl}$ está muy poco desarrollada, conclusión a la que también arriban en diversas contribuciones tanto Romanos (2010a, 2010b, 2011) como Parada $(2016,2017)$.

Asimismo, el campo disciplinar tampoco se encuentra formalizado a nivel institucional, es decir, no existe un área de evaluación específica. La categorización de docentes-investigadores que desarrollan su actividad en universidades públicas se encuentra a cargo de la Secretaría de Políticas Universitarias del Ministerio de Educación (Resolución ministerial 1543/2014). Dentro de este esquema institucional la $\mathrm{ByCl}$ no cuenta con las áreas o comisiones de pares que califican y acreditan a aspirantes, y son especialistas de otros campos quienes analizan y asignan calificaciones a los curriculum y las producciones de quienes se presentan (Liberatore, 2019). En lo que respecta a la carrera de investigación en el CONICET, es muy incipiente la inserción de investigadores del área allí, pero comienza a haber un interés por desarrollar carreras de investigación y se visualiza también como una posible fuente laboral.

En el año 2015 se lanzó la Maestría en Bibliotecología y Ciencia de la Información, producto de un acuerdo entre la UBA y la Biblioteca Nacional "Mariano Moreno" (Barber y Pisano, 2014). La misma tuvo una cohorte 2016-2017 con modalidad presencial y se discontinuó. Consistía en dos ciclos anuales que comprendían materias o cursos teóricos y teórico-prácticos, seminarios y talleres (distribuidos en 704 horas reloj, correspondientes a 44 créditos). Con respecto al egreso, no hay fuentes claras sobre la cantidad de personas que obtuvieron la titulación; aunque por informaciones procedentes de fuentes informales que se obtuvieron, fue exigua. Otra propuesta a nivel de especialización es la que ofrece la UNLP con la Especialización en Gestión de Información Científica y Tecnológica. Se comenzó a ofrecer en el año 2016 y, a partir del 2018, obtuvo el reconocimiento por parte de la Comisión Nacional de Evaluación y Acreditación Universitaria (CONEAU). Esta modalidad aún no posee egreso.

En la actualidad, existen dos nuevas propuestas llevadas adelante en la UNMDP para la formación en el nivel de posgrado, ambas en procesos de evaluación y acreditación. Se trata de una maestría en Gestión de Información Científica con orientación a las Ciencias Sociales y las Humanidades y de un doctorado en Ciencias Sociales que incluye la orientación en el campo de la Ciencia de la Información. De concretarse dichos proyectos para el 2021, la ByCl argentina completaría por primera vez en su historia todos los niveles de formación en el nivel de posgrado, comenzando por la especialización antes mencionada que se dicta en la UNLP. El desarrollo de estas instancias de posgrado se espera que contribuyan a fortalecer la investigación en el campo de la $\mathrm{ByCl}$, además de incorporar miradas innovadoras y constructivas. Un camino que seguramente supondrá varios años de espera hasta su consolidación, pero que urge iniciarlo y sostenerlo. 


\section{METODOLOGÍA}

\subsection{Abordaje teórico}

Para lograr una mejor caracterización y sistematización de la investigación desarrollada en $\mathrm{ByCl}$ en Argentina, se recuperó la producción científica generada en los últimos veinte años (periodo 2001-2020). Esta línea de abordaje empírica se basa en la idea de que a través del análisis de los productos intelectuales publicados o inéditos concebidos desde la actividad investigadora/académica se puede obtener una imagen más cercana y real del verdadero desarrollo de esta dimensión, al tiempo de observar con mayor nitidez sus particularidades y contornos. Como ya se ha mencionado, los procesos de institucionalización de la actividad académica de este campo disciplinar en nuestro país han sido complejos e incompletos, en el sentido de que no todas las carreras universitarias cuentan con infraestructura y recursos financieros para el pleno desarrollo de la actividad investigadora. Esta situación provoca, entre otras cuestiones, que no existan líneas de investigación declaradas o formales, ni tampoco un ámbito de alcance nacional desde donde se establezcan directrices o consensos en torno a los lineamientos y definición de problemáticas que encaucen los esfuerzos realizados desde los espacios de formación. A toda esta realidad, se ha de agregar que la mayoría de las carreras universitarias poseen un perfil de formación profesionalista, no académico, relegando la investigación a un segundo plano.

En este escenario, una descripción y un diagnóstico de esta área deben construirse, en principio, desde el estudio de los aportes genuinos y originales a nuestro campo que en muchos casos se encuentran invisibilizados o difundidos en canales de comunicación estrictamente locales o de circulación nacional. Así, al considerar esta perspectiva, se ha tomado como muestra de estudio la producción científica cuya autoría provenga de docentes-investigadores que integren o hayan integrado el cuerpo académico de alguna de las siete escuelas de universidades públicas del país en los últimos veinte años (2001-2020) y entendiendo por tal a las producciones publicadas o inéditas a las que se pueda acceder a su registro. En tal sentido, las unidades de análisis quedaron conformadas por los investigadores que cumplieran con las condiciones antes fijadas $(n=235)$ y los registros recuperados pertenecientes a sus producciones $(n=1530)$ dentro del periodo establecido.

Para el procesamiento de este corpus, a partir de los datos obtenidos, se han delimitado los siguientes indicadores: productividad, tipos documentales y áreas temáticas de producción. Para la distribución por áreas temáticas se ha tomado la clasificación consensuada en el ámbito de Mercosur en la reunión realizada en Belo Horizonte en el año 2016. Hubo una posterior en 2019, llevada a cabo en Asunción del Paraguay, pero la asistencia de docentes fue muy exigua y no se circularon documentos finales de dicho evento. Las áreas son, de acuerdo con la relatoría de Mirta Juana Miranda (2016), las siguientes:

- Introducción a las disciplinas y profesiones. Fundamentos teóricos;

- Organización y tratamiento de la información;

- Recursos y servicios de información;

- Gestión de la información en organizaciones;

- Tecnología de la información;

- Investigación en Bibliotecología y Ciencia de la Información y áreas afines; y 
- Prácticas e innovaciones pedagógicas.

Finalmente, el esquema de áreas tuvo que ser levemente modificado y ampliado debido a que en la práctica algunas escuelas no han actualizado sus diseños curriculares o incluyen espacios de formación vinculados a disciplinas afines como la museología o la archivología. La clasificación final utilizada fue la presentada en la tabla 1. Los datos fueron recogidos y analizados entre los meses de diciembre de 2020 y febrero de 2021.

\begin{tabular}{lc}
\hline Áreas temáticas & Abreviatura \\
\hline Teórico Metodológica/Investigación & TM \\
Organización y Recuperación de Información & OR \\
Gestión de Información & $\mathrm{GI}$ \\
Recursos y Servicios de Información & $\mathrm{RS}$ \\
Tecnología de la Información & $\mathrm{TI}$ \\
Ciencias Sociales & $\mathrm{CS}$ \\
Didáctica/Enseñanza ByCl & $\mathrm{DI}$ \\
Museos & $\mathrm{MU}$ \\
Archivos & $\mathrm{AR}$ \\
Conservación y Preservación & $\mathrm{CO}$ \\
\hline
\end{tabular}

Tabla 1. Áreas de conocimiento en ByCl (Fuente: elaboración propia).

\subsection{Fuentes de datos}

Dada la dispersión documental sobre la que se basa este estudio, teniendo en cuenta el alcance del concepto de producción científica descripto en párrafos anteriores, se seleccionó Google Académico (GA) como fuente de datos principal. Este buscador se seleccionó debido a su algoritmo de indexación, dado que posee una cobertura muy amplia de la producción generada en ámbitos académicos, por encima de otras bases de datos bibliográficas (Martín-Martín et al., 2018; Martín-Martín et al., 2020). Complementariamente, se utilizaron otras fuentes secundarias como Scielo, Redalyc, DOAJ y las bases de corriente principal Web of Science (WoS) y Scopus. Para las búsquedas en GA, WoS y Scopus se utilizó la aplicación Publish or Perish (v. 7.27) (Harzing, 2020). Finalmente, se realizó una depuración de los ítems hallados filtrando aquellos tipos documentales que no se corresponden con la actividad investigadora tales como programas de asignaturas, entrevistas, videos y entradas de blogs.

Las búsquedas se realizaron por los nombres de docentes-investigadores de las siete escuelas de universidades públicas de ByCl (UBA, UNAM, UNC, UNLAR, UNLP, UNMDP y UNNE). Exceptuando la UNLAR, por ser de reciente creación, estas son las escuelas de mayor tradición en la disciplina. Han quedado fuera de este estudio las escuelas de la UNL y UADER, dado que no fue posible acceder a datos de sus plantas docentes. Para garantizar la cobertura total del universo seleccionado, se construyó previamente un directorio de profesionales que integran o han integrado en los últimos veinte años las plantas docentes de las carreras, con indicación de las asignaturas y áreas temáticas en donde se desempeñan. Este último dato es el que se utilizó para la clasificación de la producción recuperada por 
área de origen. Para el procesamiento y cálculo de los indicadores se diseñó una base de datos ad hoc y los resultados fueron procesados y representados mediante la herramienta Microsoft Excel.

\subsection{Limitaciones}

Si bien está comprobada y documentada la capacidad de indexación de GA en el ámbito de la producción académica, es importante destacar que los resultados obtenidos se enfrentan a ciertas limitaciones. Alguna de éstas proviene de la propia herramienta, y otras, de la baja calidad de la descripción bibliográfica y/o de la estructura y formato de los documentos indexados. Un aspecto negativo en este sentido se relaciona con el bajo porcentaje de docentes que poseen un perfil en este buscador (no supera el 40\%), desde donde es posible recuperar de manera efectiva, y con un cierto control, los registros de la producción acreditada. La búsqueda individual de aquellos investigadores que no poseen este perfil se vio enfrentada en algunos casos a las dificultades de verificación de la autenticidad de ciertos documentos, certificación de autorías y de las fuentes de difusión. En todos los casos se realizaron procesos de filtrado manual de los datos consignados. Teniendo en cuenta estas particularidades, consideramos que el porcentaje de la producción intelectual que no ha podido ser relevada es muy bajo y, por tanto, los resultados obtenidos representan con bastante fidelidad el dominio analizado.

\section{RESULTADOS Y DISCUSIÓN}

Para abordar con mayor precisión y riqueza las contribuciones recuperadas se diseñó una clasificación suficientemente amplia de los tipos documentales recuperados (Figura 1), la cual permite reflejar las múltiples formas de difusión de los productos intelectuales.

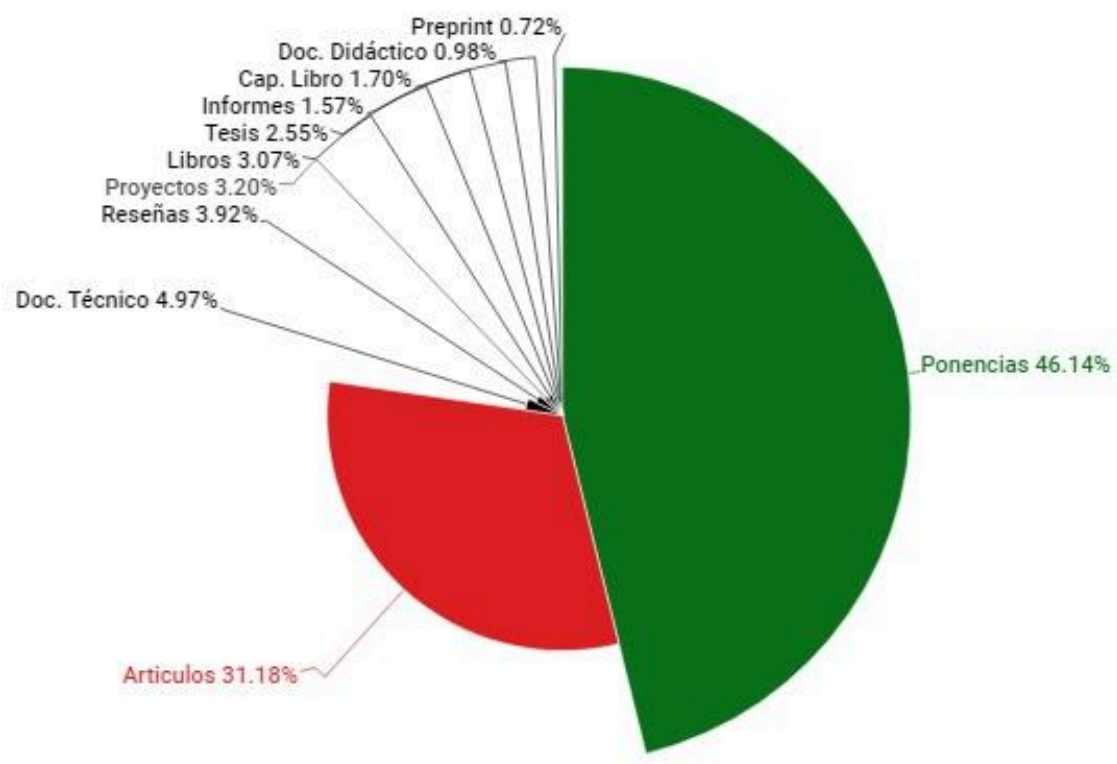

Figura 1. Tipologías documentales recuperadas $(n=1.530)$ expresadas en porcentajes (Fuente: elaboración propia).

Como era de esperar, las dos principales formas de comunicación de la actividad investigadora son canalizadas a través de las reuniones y artículos científicos ( $46 \%$ y $31 \%$ respectivamente), lo que constituye el $77 \%$ del corpus analizado. Si se suman los libros y 
capítulos de libro, se arriba al hecho de que el $82 \%$ de la producción científica de $\mathrm{ByCl}$ recuperada se difunde por medio de canales formales. La preeminencia de las ponencias en reuniones científicas constituye el dato más relevante develando un patrón de comunicación preponderante. Esta situación se debe en gran parte a que la oferta de jornadas y congresos a nivel local, tanto de la disciplina como de áreas conexas, es muy superior al del número de títulos nacionales de revistas de $\mathrm{ByCl}$ (sólo tres publicaciones académicas); que se constituyen como los principales espacios seleccionados para la publicación de artículos.

Al analizar en particular estas dos dimensiones (ponencias y artículos) se observa que, en el caso de los artículos, el $77 \%$ fue publicado en revistas del área de la $\mathrm{ByCl}$, mientras que el restante $33 \%$ se publicó en otras áreas disciplinares, mayormente en el campo de las ciencias sociales y humanidades. Dentro del grupo de revistas de $\mathrm{ByCl}$, casi la mitad de los trabajos (46,5\%) se publicaron en títulos argentinos con un notable protagonismo de las fuentes con más tradición académica en este ámbito, Información, cultura y sociedad (UBA) y Palabra clave (UNLP), concentrando ambas el $36 \%$ de las publicaciones. Se observa en este último aspecto un fenómeno común en las publicaciones editadas por instituciones universitarias, en el sentido de la importante presencia de autores cuya filiación proviene del mismo ámbito. El resto de los títulos observados del campo de la ByCl tienen su origen mayoritariamente en países latinoamericanos (20,5\%), destacándose Brasil y Colombia, y una presencia relativa de revistas españolas (6\%). Sólo el $4 \%$ de los artículos ha sido publicado en inglés. Para el caso de las ponencias se aprecia una situación similar ya que el $62 \%$ se han presentado en reuniones científicas del campo disciplinar, con preferencia en ámbitos nacionales (49\%). El 38\% restante está acreditado en jornadas y congresos de otros campos disciplinares, mayoritariamente de las ciencias sociales y humanidades.

En el análisis global de los dos formatos documentales predominantes emerge una singularidad, dado que aproximadamente un tercio de la producción científica circula por espacios disciplinares que no son propios. No constituye una novedad el carácter interdisciplinario que conlleva el abordaje de las problemáticas de investigación en nuestro campo y en nuestro tiempo, aunque es posible advertir que este fenómeno adquiere, a partir de la observación de los resultados, numerosas aristas. En principio, es importante decir que la "interdisciplinariedad" se da en varios sentidos y formas y que existe un predominio en los procesos de interacción y vinculación con disciplinas de las ciencias sociales. Pueden observarse transferencias de conocimiento tanto "desde" como "hacia" otros campos a partir de metodologías y enfoques teóricos. En particular, hay buenos ejemplos de este fenómeno de intercambio en al área de la gestión de información, los servicios y recursos y muy particularmente en el terreno de los estudios métricos de la información. Esta realidad, en tanto, no resulta azarosa. Existe un primer argumento más general y de difícil medición relacionado con una maduración y un número creciente de investigadores que ha propiciado la creación y ampliación de espacios y líneas de desarrollo. Asimismo, dos factores muy concretos provenientes de la actividad tanto de la docencia como de la investigación: la inserción de investigadores en grupos de otras disciplinas por falta de las condiciones necesarias para crear espacios propios, y la existencia de un porcentaje significativo, en términos generales, de profesionales de otras disciplinas que integran los planteles docentes de las carreas de ByCl.

Al observar la distribución temporal de la producción científica en los últimos veinte años puede advertirse que su evolución es muy irregular, con notables fluctuaciones interanuales expresadas estadísticamente a partir de una tendencia de tipo polinómica (Figura 2). Si bien los valores tienden a incrementarse con el paso del tiempo, con excepción de los últimos tres años, los avances y retrocesos en términos de la productividad son importantes. Esta particularidad puede deberse a varios factores. Una primera cuestión, más general, 
puede relacionarse con una falta de maduración del campo disciplinar en el sentido de no constituirse todavía como un área de investigación plenamente institucionalizada y, por lo tanto, susceptible a vaivenes en el rendimiento y productividad de sus espacios de investigación propios. Un segundo aspecto, más reconocible, es el diferencial existente entre las escuelas analizadas desde el punto de vista de sus trayectorias y potencialidad de desarrollo lo que provoca las distorsiones observadas en una análisis global de sus producciones (Figura 3). En términos de la consolidación de la actividad investigadora y de los recursos humanos formados para esta dimensión UNLP, UBA y UNMDP se constituyen como espacios de liderazgo. Las tres escuelas concentran el $82 \%$ de la producción recuperada durante el período estudiado. No obstante, es probable que la productividad del 2020 haya sido afectada por la pandemia de COVID-19, especialmente por la cancelación de muchos eventos científicos y académicos; aunque esta justificación no tendría validez para los dos años anteriores, en los que otros factores pudieron haber impactado en ese resultado.

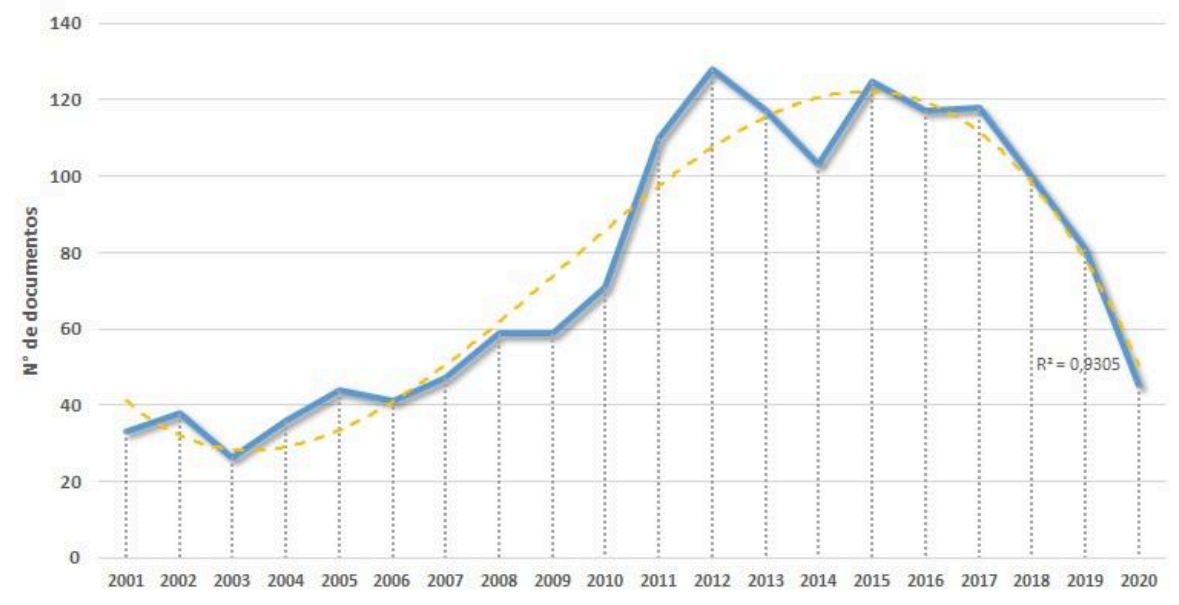

Figura 2. Distribución temporal de la producción científica (2001-2020) (Fuente: elaboración propia).

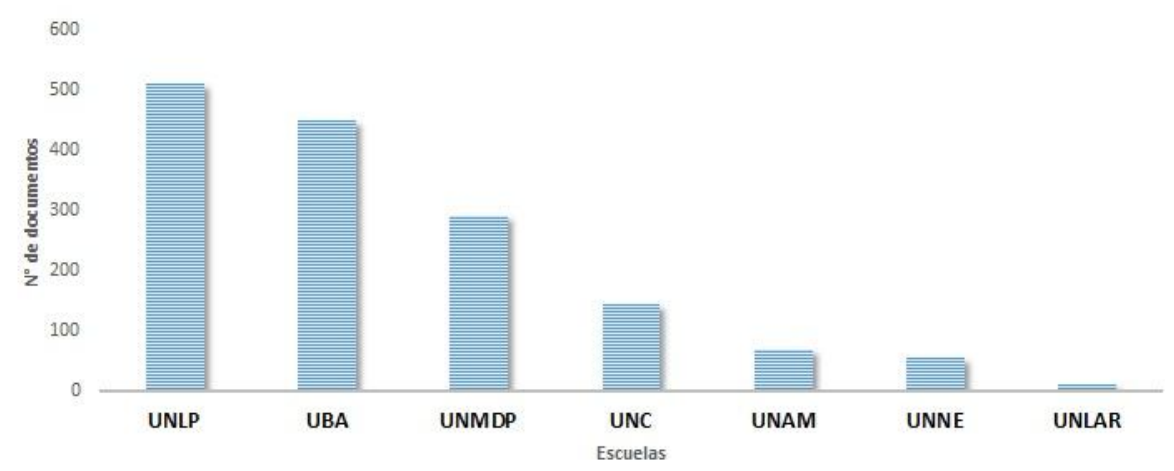

Figura 3. Distribución de la producción científica discriminada por escuela (Fuente: elaboración propia).

El análisis de la producción por áreas temáticas se llevó a cabo con cierta complejidad debido a dos cuestiones que inciden particularmente en esta dimensión. Por un lado, hay profesionales que suelen desempeñar sus funciones de docencia e investigación en más 
de un área, por lo que en muchos casos debió recurrirse a una cuenta fraccionaria en la asignación de sus producciones intelectuales. Por otro, existen temáticas que son abordadas desde varios puntos de vista en lo que se traduce en una participación autoral proveniente de áreas temáticas diferentes. Aun así, y tomando en cuenta la configuración propuesta en el apartado metodológico, pudo establecerse una representación bastante ajustada a la realidad.

En la distribución global del corpus analizado, los aspectos relacionados a los abordajes teóricos de la disciplina y las cuestiones relativas al estudio y aplicación de las metodologías utilizadas en la práctica de investigación (TM) son los que acreditan la mayor producción (Figura 4). Esta área representa el $31 \%$ del total calculado. No obstante, este es al mismo tiempo el espacio que presenta los contornos más difusos frente a las demás áreas, en el sentido de la transversalidad de sus contenidos y enfoques, donde se pueden apreciar los rasgos epistemológicos preponderantes en el desarrollo de la disciplina en nuestro país.

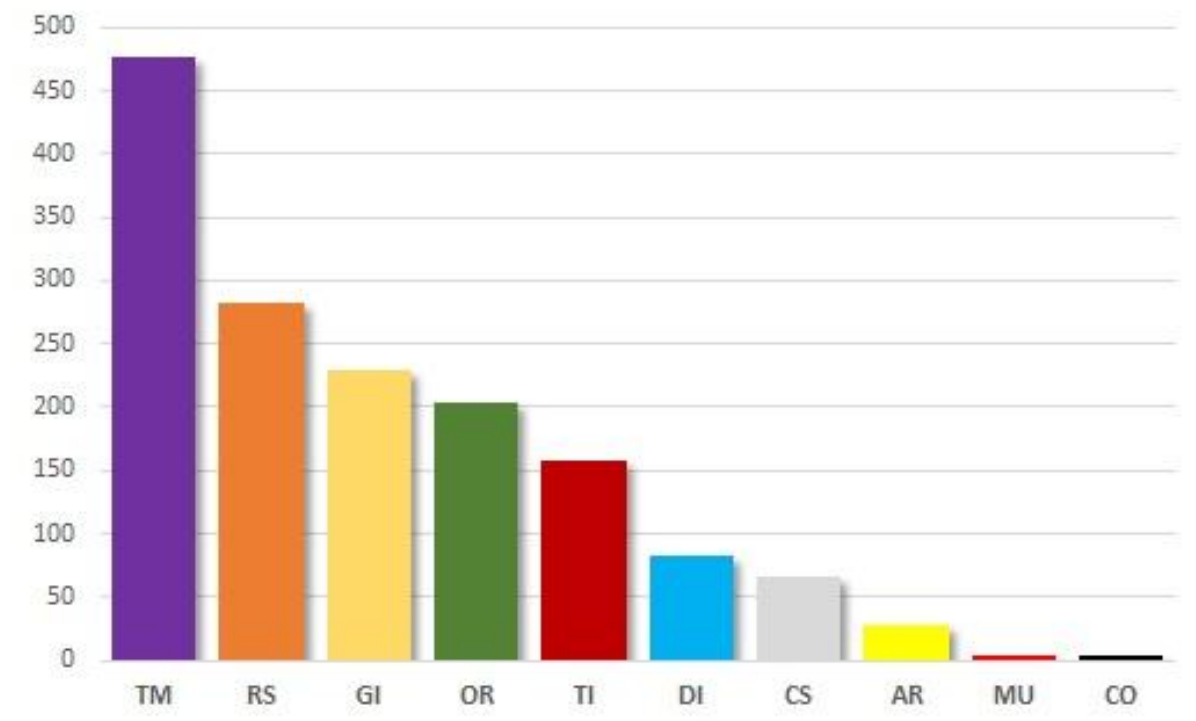

Figura 4. Distribución de la producción por área temática (Fuente: elaboración propia).

Le siguen en importancia las tres áreas troncales de la $\mathrm{ByCl}$, como son los recursos y servicios de información (RS), la gestión de información (GI) y la organización y representación del conocimiento (OR), respectivamente. Las tecnologías de la información (TI) aparecen en el orden de producción aunque esta es también un área de difícil demarcación en términos de la dependencia y articulación de sus contenidos al de las áreas troncales. Las cuestiones relacionadas a la enseñanza de la $\mathrm{ByCl}$ y sus implicancias pedagógicas y didácticas (DI) emergen como un espacio de creciente interés en casi todas las carreras; aunque sólo existen tres escuelas que ofrecen la titulación en el profesorado (UBA, UNMDP y UNLP). En séptimo lugar se ubica un ámbito de producción denominado ciencias sociales (CS) que no es estrictamente temático, sino más bien multidisciplinar, que ha sido definido para poder agrupar todas aquellas contribuciones que provienen de investigadores que aportan estudios desde ámbitos de formación ajenos a la $\mathrm{ByCl}$ y que, como se explicaba en párrafos anteriores, están a cargo de asignaturas en los planes de estudios de las carreras. Aquí están englobados trabajos ligados a las ciencias sociales y humanidades como: lectura, literatura infantil, historia, educación, metodología de investigación, estadística, administración y gestión de organizaciones, entre otros. Finalmente, se han observado numerosos trabajos unidos a disciplinas conexas que se integran desde el paradigma de las ciencias de la información, como son la archivología (AR), 
la museología (MU) y la conservación (CO), en particular en aquellas carreras que ofrecen títulos con orientación en alguno de estos campos (ej.: UNNE, UBA y UNLAR), o bien poseen asignaturas específicas en estas áreas (ej.: UNMDP).

Cuando se analiza el desempeño relativo de las áreas por escuela, se observa una serie de características ligadas íntimamente a una serie de factores relacionados con las trayectorias académicas de los docentes que componen las carreras, la historia y antigüedad de los Departamentos, así como los perfiles de las instituciones universitarias donde están insertados (Figura 5).

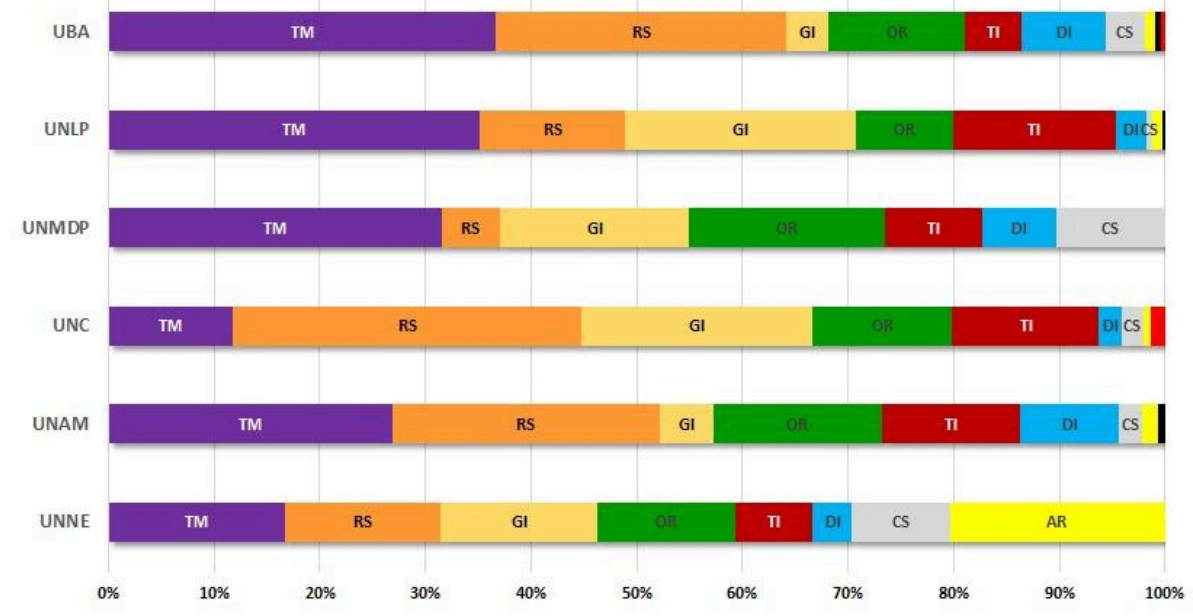

Figura 5. Distribución de la producción científica por escuela y área temática (Fuente: elaboración propia).

En esta representación se ha excluido a la UNLAR por ofrecer una carrera de muy reciente creación y que no admite comparación con las demás de mayor consolidación. En este sentido, la primera cuestión a resaltar es que en las tres escuelas que lideran la producción científica (ej.: UNLP, UBA y UNMDP) es donde se observa el mayor desarrollo del área teórico-metodológica/investigación (TM), superando en más de un tercio al total de su producción en los tres casos. Esto nos lleva a pensar en la existencia de una relación con la mayor capacidad de infraestructura y recursos humanos formados en la investigación. Por fuera de esta particularidad, puede apreciarse que mientras algunas escuelas muestran un desarrollo con un cierto equilibrio en términos de sus volúmenes de producción de las áreas propias de $\mathrm{ByCl}$ (ej.: UNLP, UNNE), hay otras con predominio de unas áreas sobre otras, como es el caso de recursos y servicios (ej.: UBA, UNC, UNAM y UNMDP) y organización y representación del conocimiento (ej.: UNMDP) o archivología (ej.: UNNE).

Más allá de esta descripción esquemática, puede realizarse un nivel de análisis más específico desde el punto de vista de las temáticas de investigación prevalentes en algunas de las escuelas, y que revelan un grado de especialización mayor en sus investigadores y en la constitución de frentes de actividad científica sistemática. Tal es el caso de los estudios métricos de información y la evaluación científica (ej.: UNLP y UNMDP), bibliotecas escolares y promoción de la lectura (ej.: UNMDP), acceso abierto en la producción científica (ej.: UNLP), historia de las bibliotecas y el libro (ej.: UNLP, UBA), teoría de la ByCl (ej.: UBA), sistemas de organización del conocimiento (ej: UNLP, UNMDP), descripción bibliográfica (ej.: UNC, UBA), evaluación de colecciones y servicios (ej.: UNLP y UNC) y bibliotecología social (ej.: UBA). 


\section{CONCLUSIONES}

Hace dos décadas atrás, Romanos (2001) advertía la ausencia de una masa crítica de investigadores que dirigieran diseños experimentales, cuasi experimentales, descriptivos o de otros tipos en $\mathrm{ByCl}$. Esta realidad, teniendo en cuenta los progresos experimentados en el campo que aquí se han descripto, varió bastante de aquel panorama presentado por la autora, aunque no rotundamente. La cuestión del reconocimiento de ByCl en el sistema de investigación, tanto en el CONICET como en el ámbito universitario nacional, se torna en un tema central para la disciplina. Se han efectuado avances, más aún resta un largo camino por transitar en tal sentido.

Si bien desde lo formativo hay una variedad de carreras en varios lugares del país, tanto a nivel público como privado, los aportes provenientes de cada una de las universidades son disímiles y es necesario que la investigación comience a consolidarse como uno de los pilares esenciales, además de la docencia y la extensión. Dicho proceso requiere de reflexiones en la búsqueda de las claves que expliquen el presente que se experimenta, una trayectoria que aquí se pretendió describir en los últimos veinte años, pero que data de mucho antes. Asimismo, exige de políticas institucionales que permitan superar el escenario planteado en pos de un incremento de líneas de estudio, investigadores y recursos necesarios para llevar adelante tamaña tarea.

Un aspecto positivo es la formalización en la comunicación de los resultados de investigación en ponencias o artículos de revistas, que atraviesan procesos de evaluación y validación por pares también. Otro que se puede destacar es la producción en todos los ejes considerados esenciales del campo de la $\mathrm{ByCl}$, más allá de los porcentajes correspondientes a estos últimos veinte años que consolidan más a unos que a otros. La edición de tres revistas específicas también demuestra un creciente interés de las escuelas por aportar a la $\mathrm{ByCl}$ en el país, con el esfuerzo y dedicación que implica mantener una publicación científica de acceso abierto.

Tal como señala Parada (2017) en su editorial con motivo del $50^{\circ}$ aniversario del INIBI-UBA:

(...) creo imprescindible el intento de superar y abandonar, con franca decisión, nuestros relatos endógenos, sean de índole temática, de características prácticas, de dependencia de las fuentes, de ausencia de polémica, o de pasión por lo sistémico. Intentar, inequívocamente, refundarnos fuera de nuestros complejos de endogamia e instalarnos en otras territorialidades para expandir nuestra identidad (p. 10).

Las palabras antes citadas en parte responden a la realidad abordada en este trabajo, aunque ha de destacarse que resta mucho camino por recorrer en el fortalecimiento de la investigación en cada ámbito universitario, en cuanto a líneas de trabajo específicas de $\mathrm{ByCl}$, formación de investigadores, producción intelectual, comunicación académica y científica, entre otras aristas. Un crítico equilibrio entre interdisciplinariedad y fortalecimiento del campo, una postura más beligerante por obtener los reconocimientos y respaldos institucionales que favorezcan el crecimiento de la disciplina, sumado a un aporte sostenido basado en el estudio y la investigación en los equipos que con esfuerzo se han conformado en cada universidad. Estos son los desafíos para sortear en los próximos años, además de mantener abiertos los diálogos entre formación de grado, posgrado e investigación. 


\section{Notas}

${ }^{1}$ http://inibi.institutos.filo.uba.ar/

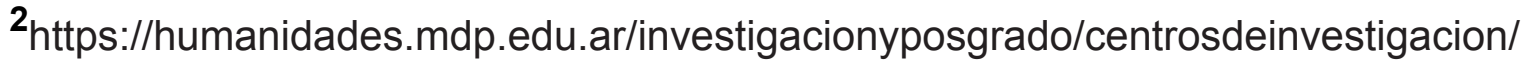
centro-de-estudios-en-ciencia-de-la-informacion-y-documentacion-cecid/

${ }^{3}$ Ciclo de charlas del INIBI 2020: https://www.youtube.com/ playlist?list=PLR6Z3NgYJX9LwsAvQjHbzPwLILq9crMHs; Ciclo de charlas del INIBI 2021: https://www.youtube.com/playlist?list=PLR6Z3NgYJX9L7Pm1UFjUHPIMICShjR2Vk

\section{Declaración de contribución}

Conceptualización, Curación de Datos, Análisis Formal, Investigación, Metodología, Recursos, Validación, Visualización, Redacción-Borrador Original, Redacción-Revisión y Edición: María Cecilia Corda y Gustavo Liberatore.

\section{Declaración de conflicto de interés}

Declaramos que no mantenemos relaciones personales, académicas o económicas con terceras personas que hayan influido en el contenido del trabajo de investigación enviado para su publicación.

\section{Declaración de consentimiento de datos}

Declaramos que los datos generados durante el desarrollo de esta investigación se han incluido en el manuscrito enviado para su publicación.

\section{REFERENCIAS}

Araújo, C.A.A.; \& Valentim, M. L. P. (2019). A ciência da informação no Brasil: mapeamento da pesquisa e cenário institucional. Bibliotecas. Anales de investigación, 15(2), 232-259. Recuperado de http://revistas.bnjm.cu/index.php/BAl/article/view/131/130

Barber, E.E. (2007, octubre). Evolución de los Encuentros de las Escuelas de Bibliotecología y Ciencia de la Información de los países del Mercosur (1996-2007). En Jornada por los 60 años de la carrera de Bibliotecología en la Universidad Nacional de La Plata, UNLP-FAHCE La Plata, Argentina. Recuperado de https://www.bn.gov.ar/resources/conferences/pdfs/ ponencia_barber_formacion.pdf

Barber, E.E.; \& Pisano, S. (2014). Maestría en Bibliotecología y Ciencia de la Información. Buenos Aires: s. n. Recuperado de https://www.bn.gov.ar/resources/conferences/pdfs/ ponencia-Barber-Pisano.pdf

Bosch, M.A. (2015, agosto). Investigación en Bibliotecología y Ciencias de la Información: hacia los Laboratorios de Información. Perspectivas desde el Centro Argentino de Información Científica y Tecnológica. En Segundas Jornadas Internas de Investigación en Bibliotecología Jornadas en Homenaje a la trayectoria de la Prof. Aurora "Tuti" Chiriello, Departamento de Documentación, Facultad de Humanidades, UNMdP, Argentina. Recuperado de http://humadoc.mdp.edu.ar:8080/xmlui/handle/123456789/436 
González, C.M.; \& Miguel, S.E. (2010, diciembre). Composición y dinámica de los grupos de investigación del Departamento de Bibliotecología de la Universidad Nacional de La Plata en el periodo 2000-2009. En I Jornadas de Intercambio y Reflexión acerca de la Investigación en Bibliotecología, UNLP-FAHCE, La Plata, Argentina. Recuperado de http://www.memoria.fahce.unlp.edu.ar/library?a=d\&c=eventos\&d=Jev775

Harzing, A. (2020).Publish or Perish. Recuperado de https://harzing.com/

Liberatore, G. (2002, septiembre). Políticas de investigación: acciones dirigidas a la integración de la graduación y el postgrado. En VI Encuentro de Directores y V de Docentes de Escuelas de Bibliotecología y Ciencia de la Información del MERCOSUR, Londrina, Brasil.

Liberatore, G. (2011). Niveles de institucionalización de la bibliotecología y ciencia de la información en Argentina: una aproximación desde un enfoque empírico. Perspectivas em gestão \& conhecimento, 1(1), 150-162. Recuperado de https://periodicos.ufpb.br/ojs2/ index.php/pgc/article/view/9802

Liberatore, G., Sleimen, S., Coringrato, M., Sanllorenti, A. M., Vuotto, A., Fernández, G., ... \& Saya, J. (2015, octubre). Patrones de publicación de los investigadores de la Fac. de Humanidades de la UNMdP durante el período 2009-2013: análisis de autoría y fuentes. En Segundas Jornadas Internas de Investigación en Bibliotecología Jornadas en Homenaje a la trayectoria de la Prof. Aurora "Tuti" Chiriello, UNMDP, Mar del Plata, Argentina. Recuperado de http://humadoc.mdp.edu.ar:8080/bitstream/handle/123456789/434/ Ponencia-Analisis-de-la-actividad-cientifica-jiib-2015.pdf?sequence=1

Liberatore, G. (2019, diciembre). Visión actual del profesional de la Bibliotecología y Ciencia de la Información en la docencia y la investigación. En 2das Jornadas de Docentes Investigadores Universitarios en Ciencia de la Información, UNMDP, Mar del Plata, Argentina. Recuperado de http://humadoc.mdp.edu.ar:8080/handle/123456789/ 850 ?show=full

Martín-Martín, A., Orduna-Malea, E., Thelwall, M., \& Delgado López-Cózar, E. (2018). Google Scholar, Web of Science, and Scopus: a systematic comparison of citations in 252 subject categories. Journal of informetrics, 12(4), 1160-1177. https://doi.org/10.1016/ j.joi.2018.09.002

Martín-Martín, A.; Orduna-Malea, E.; Thelwall, M., \& Delgado López-Cózar, E. (2020). Google Scholar, Microsoft Academic, Scopus, Dimensions, Web of Science, and OpenCitations' COCl: a multidisciplinary comparison of coverage via citations. Scientometrics, 126(1), 871-906. https://doi.org/10.1007/s11192-020-03690-4

Miguel, S.E. (2009). Oportunidades y desafíos actuales de la investigación en Bibliotecología y Ciencia de la Información. El caso del Departamento de Bibliotecología de la Universidad Nacional de La Plata, Argentina. Información, cultura y sociedad, 21, 51-67. https://doi.org/ 10.34096/ics.i21.795

Miguel, S.E. (2015, octubre). Mirarnos a nosotros mismos: consolidando espacios para el debate y la reflexión sobre la investigación en Bibliotecología y Ciencia de la Información. En IV Jornadas de Intercambio y Reflexión acerca de la Investigación en Bibliotecología, UNLP-FAHCE, La Plata, Argentina. Recuperado de http://www.memoria.fahce.unlp.edu.ar/ library?a $=\mathrm{d} \& \mathrm{c}=$ eventos $\& \mathrm{~d}=\mathrm{Jev} 5287$ 
Miguel, S.E., González, C.M., \& Archuby, C. (2010, diciembre). El desarrollo de la línea de investigación de estudios métricos de la información en el Departamento de Bibliotecología de la UNLP: facetas y perspectivas. En I Jornadas de Intercambio y Reflexión acerca de la Investigación en Bibliotecología, UNLP-FAHCE, La Plata, Argentina. Recuperado de http://www.memoria.fahce.unlp.edu.ar/library?a=d\&c=eventos\&d=Jev758

Miguel, S.E., Chinchilla-Rodríguez, Z., \& González, C.M. (2010, diciembre). Análisis de la composición y dinámica de grupos de investigación en perspectiva comparada input-ouput: estudio de caso en Bibliotecología y Ciencia de la Información. En VI Jornadas de Sociología de la UNLP, La Plata, Argentina. Recuperado de http://www.memoria.fahce.unlp.edu.ar/ library? $a=d \& c=e v e n t o s \& d=J e v 3943$

Miguel, S.E., Hidalgo, M., Stubbs, E., \& Planas, J. A. (2012, octubre). Reflexiones y propuestas en torno a la formación y la práctica de la investigación en Bibliotecología y Ciencia de la Información. En VIII Encuentro de Docentes de Escuelas de Bibliotecología y Ciencia de la Información del Mercosur, Montevideo, Uruguay. Recuperado de http://www.memoria.fahce.unlp.edu.ar/library?a=d\&c=eventos\&d=Jev3945

Miguel, S.E., \& Dimitri, P.J. (2013). La investigación en bibliometría en la Argentina: quiénes son y qué producen los autores argentinos que realizan estudios bibliométricos. Información, cultura y sociedad, 29,117-138. https://doi.org/10.34096/ics.i29.677

Miguel, S.E.; Salomoni, D., \& Hidalgo, M. (2014, octubre). La formación en investigación en las carreras de grado de Bibliotecología y Ciencia de la Información en universidades nacionales argentinas. En IX Encuentro de Docentes de Escuelas de Bibliotecología y Ciencia de la Información del Mercosur, Ciudad Autónoma de Buenos Aires, Argentina. Recuperado de http://www.memoria.fahce.unlp.edu.ar/library?a=d\&c=eventos\&d=Jev7890

Miranda, J.M. (2017). XI Encuentro de Directores y X de Docentes de Escuelas de Bibliotecología y Ciencia de la Información del MERCOSUR: relatoría. Información, cultura y sociedad, 36, 123-137. https://doi.org/10.34096/ics.i36.3553

Parada, A.E. (2016). Escritura y Bibliotecología/Ciencia de la información. Información, cultura y sociedad, 35, 5-10. https://doi.org/10.34096/ics.i35.2881

Parada, A.E. (2017). Endogamia y Bibliotecología/Ciencia de la Información. Información, cultura y sociedad, 36, 5-10. https://doi.org/10.34096/ics.i36.3551

Parada, A.E. (2019). Homenaje al INIBI en el cincuentenario de su creación (1967-2017). Información, cultura y sociedad, 41, 201-204. https://doi.org/10.34096/ics.i41.7025

Pinheiro, L.V.R., \& Loureiro, J.M.M. (2004, abril). Políticas públicas de C\&T, ICT e de Pós-Graduação em abordagem histórico-cultural da ciência da informação no Brasil. En CINFORM - Encontro Nacional de Ciência da Informação, Salvador da Bahia, Brasil. Recuperado de http://www.cinform-anteriores.ufba.br/v_anais/artigos/vaniajose.html

Romanos, S. (2001). Investigación y práctica profesional. Información, cultura y sociedad, 4, 6-8. https://doi.org/10.34096/ics.i4.1008

Romanos, S. (2010a). Los desafíos de la investigación bibliotecológica en la Argentina. Información, cultura y sociedad, 22, 5-10. https://doi.org/10.34096/ics.i22.761 
Romanos, S. (2010b). Itinerarios posibles de producción de conocimiento en bibliotecología/ ciencia de la información. Información, cultura y sociedad, 22, 79-98. https://doi.org/ 10.34096/ics.i22.764

Romanos, S., Contardi, S., \& Giunti, G. (2010). Estrategias de difusión del conocimiento de los investigadores en la Facultad de Filosofía y Letras de la Universidad de Buenos Aires. Información, cultura y sociedad, 23, 31.62. https://doi.org/10.34096/ics.i23.745

Universidad de Buenos Aires. (2015, noviembre). $1^{\circ}$ Jornadas de Docentes Investigadores Universitarios en Ciencia de la Información, Buenos Aires, Argentina. Recuperado de http://www.fhycs.unam.edu.ar/DUCl/jornadas-anteriores/1-jornada/

Universidad Nacional de Mar del Plata. (2018, noviembre). $2^{\circ}$ Jornadas de Docentes Investigadores Universitarios en Ciencia de la Información, Mar del Plata, Argentina. En Desafíos de los formadores en la era digital. Ciudad Autónoma de Buenos Aires: Universidad de Buenos Aires. Instituto de Investigaciones Bibliotecológicas. Recuperado de http://www.fhycs.unam.edu.ar/DUCl/jornadas-anteriores/2-jornada/

Universidad Nacional de Misiones. (2020). Jornadas Nacionales de Docentes e Investigadores Universitarios en Ciencia de la Información, (3ra: 11 y 12 de Septiembre 2019 Posadas, Misiones). En Investigación y ámbitos laborales en ciencia de la información: debates y perspectivas en escenarios complejos y cambiantes. Posadas: UNAM. Recuperado de https://rid.unam.edu.ar/handle/20.500.12219/2812 